\title{
Investigation of the Validity of the Universal Scaling Law on Linear Chains of Silver Nanoparticles
}

\author{
Mohammed Alsawafta, ${ }^{1}$ Mamoun Wahbeh, ${ }^{2}$ and Vo-Van Truong ${ }^{2}$ \\ ${ }^{1}$ College of Art and Science, American University of Kuwait, 13034 Safat, Kuwait \\ ${ }^{2}$ Department of Physics, Concordia University, Montréal, QC, Canada H4B 1R6 \\ Correspondence should be addressed to Mohammed Alsawafta; malsawafta@auk.edu.kw
}

Received 14 December 2014; Accepted 4 February 2015

Academic Editor: Zhida Xu

Copyright (C) 2015 Mohammed Alsawafta et al. This is an open access article distributed under the Creative Commons Attribution License, which permits unrestricted use, distribution, and reproduction in any medium, provided the original work is properly cited.

\begin{abstract}
Due to the wide range of variation in the plasmonic characteristics of the metallic nanoparticles arranged in linear arrays, the optical spectra of these arrays provide a powerful platform for spectroscopic studies and biosensing applications. Due to the coupling effect between the interacting nanoparticles, the excited resonance mode is shifted with the interparticle separation. The change in the resonance energy of the coupled mode is expressed by the fractional plasmon shift which would normally follow a universal scaling behavior. Such a universal law has been successfully applied on a system of dimers under parallel polarization. It has been found that the plasmon shift decays exponentially over interparticle spacing. The decay length is independent of both the nanoparticle and dielectric properties of the surrounding medium. In this paper, the discrete dipole approximation (DDA) is used to examine the validity of extending the universal scaling law to linear chains of several interacting nanoparticles embedded in various host media for both parallel and perpendicular polarizations. Our calculations reveal that the decay length of both the coupled longitudinal mode (LM) and transverse modes (TM) is strongly dependent on the refractive index of the surrounding medium $\left(n_{m}\right)$. The decay constant of the LM is linearly proportional to $n_{m}$ while the corresponding constant of the TM decays exponentially with $n_{m}$. Upon changing the nanoparticle size, the change in the peak position of the LM decreases exponentially with the interparticle separation and hence, it obeys the universal law. The sensitivity of coupled LM to the nanoparticle size is more pronounced at both smaller nanoparticle sizes and separations. The sensitivity of the coupled TM to the nanoparticle size on the other hand changes linearly with the separation and therefore, the universal law does not apply in the case of the excited TM.
\end{abstract}

\section{Introduction}

Transition metal nanoparticles have attracted considerable attention due to their unique electrical, optical, chemical, and magnetic properties as compared to their bulk counterparts $[1,2]$. Noble-metal nanoparticles of a size smaller than the incident wavelength can effectively scatter and selectively absorb light at a certain wavelength in both visible and nearinfrared regions $[3,4]$. The fascinating optical properties of the metallic nanostructures originate from the excitation of the localized surface plasmon resonance (LSPR) $[5,6]$. Due to the enhancement of the local field in the vicinity of the nanoparticles (hot spots), they are used in both biosensing $[7,8]$ and Surface Enhanced Raman Scattering (SERS) applications $[9,10]$.
Well-defined metallic nanoparticles arranged in several dimensional arrays provide an interesting opportunity to tune their optical properties over a wide range of optical parameters [11-13]. Periodic structures of nanoparticles can lead to a significant enhancement of the local electromagnetic field, which can be used to improve detection and characterization capabilities down to single-molecule level $[13,14]$. Finite chains of metallic nanoparticles are considered as energy guides because of the possibility to transport their plasmonic energies along the chain axis $[11,13,14]$. The tuned plasmonic coupling between the interacting nanoparticles is an efficient way to distribute and direct the coupled energy through the plasmonic elements. In order to optimize the coupled plasmonic energy, one needs to understand the effect of various parameters on the collective plasmonic resonances. 
These parameters are the interparticle separation $(D)$, the number of the interacting nanoparticles $(N)$, the polarization states of the incident light, and the refractive index of the host media $\left(n_{m}\right)$ where the nanoparticles are embedded. Depending on the spacing between the nanoparticles, two regimes of plasmonic coupling are considered: (i) when the nanoparticles are arranged in close-packed configurations, they interact via their near-fields and this leads to either a red- or blue-shift of the plasmonic band depending on the type of the incident light [11]. In general, this interaction results in enhanced LSPR and sensing capabilities of SERS. (ii) If the nanoparticles are further displaced by a distance comparable to the incident wavelength, they interact through their radiative fields $[11,15,16]$. These far-field couplings are important for the plasmon enhanced fluorescence of the adsorbed molecules on the nanoparticles surface [11].

The dependency of the plasmon coupling parameters on the interparticle spacing was experimentally studied for nanoparticles of different shapes arranged in various configurations. These nanoparticles were either a dimer of nanodiscs or single-sized spherical nanoparticles arranged in 1D array of different sizes [17]. These nanoparticles were embedded in host media of constant refractive index. No study is reported yet on the effect of the nanoparticle size and the dielectric properties of the host medium on the coupled plasmon resonance for linear chains composed of several plasmonic elements. It has been found that the coupled plasmon resonance wavelength of a dimer system shifts exponentially with the interparticle separation. This shift was expressed by the fractional plasmon shift leading to a universal scaling behaviour $[17,18]$. The universality relies on the fact that the decay constant is independent of the nanoparticle size, shape, metal type, and host medium. Consequently, the proposed "plasmon ruler equation" $[17,18]$ was used to evaluate the spacing between a pair of nanoparticles from their measured plasmon shift. The universal scaling behaviour has been successfully applied to a trimer of nanospheres [19]. To the best of our knowledge, no study is reported on the validity of the scaling behavior for a linear chain of nanoparticles composed of several plasmonic elements.

The first purpose of this paper is to better understand and provide a full picture of the effect of several parameters on the near-field couplings. The parameters include the nanoparticle size, chain size and dielectric properties of the surrounding media at different polarization angles. The second purpose is to examine the validity of extending the universal scaling law to a linear chain that consists of several identical silver nanospheres. We believe that the results of the current study will provide a pathway to design nanoparticle chains for many technological applications. In order to perform the required calculations, the absorption spectra of linear silver chains consisting of spherical nanoparticles were simulated by using the discrete dipole approximation (DDA) method [20-22].

\section{The Discrete Dipole Approximation (DDA)}

The DDA method is a numerical approximation used to solve Maxwell's equations of the scattering problem of electromagnetic waves by metallic nanoparticles. Based on the induced electric dipole moment in the nanoparticles, DDA is used to calculate their optical cross-sections of different shapes and sizes in complex surrounding media. It involves replacing each nanoparticle by a three-dimensional array of polarizable points arranged in a cubic configuration whose side length is equal to the interdipole spacing. The optical properties of the nanoparticles are determined by three factors: (1) the incident wavelength, (2) the polarizability of the nanoparticles, and (3) the mutual interaction between both dipoles within the same nanoparticle and other dipoles in the nearby nanoparticles. The mathematical formulation of DDA is beyond the scope of the current study and is fully described elsewhere [2022 ]. DDA is tolerant regarding the target geometry and size. The only limitation to be considered is that the interdipole separation should be smaller than the incident wavelength and any other structural parameters. The accuracy of DDA is widely accepted when a large number of dipoles are used to properly mimic the geometrical parameters of the target. In this study, the desired output of DDA is the absorption cross-section of the nanoparticle, normalized to its geometrical cross-section, which yields the corresponding efficiency $\left(Q_{\mathrm{abs}}\right)$.

\section{Target Geometry and Orientation}

The target under investigation is represented by $1 \mathrm{D}$ array of $N$ silver nanoparticles of similar polarizabilities. The selected nanoparticles are spherical in shape, different in size, placed at various separations $(D)$, and embedded in different host media. $D$ is the border-to-border distance between the nearest-neighbor nanoparticles and it is usually defined in terms of the sphere radius. The optical spectra of manybody interaction problems are calculated by using the DDA method.

The nanoparticle chains are irradiated with an electromagnetic plane wave. The structure and the orientation of the chains relative to the incident field are shown in Figure 1. The plane of incidence is set to be the $x-y$ plane and the incident electric field $(E)$ is aligned along the $y$-axis. The linearly polarized ( $p$-polarized) incident light determines the polarization angle $(\varphi)$ between $E$ and the chain axis. At oblique angles, the incident electric field has two components: one parallel to the chain axis and the other one oriented along the $y-z$ plane. In this case, the observation of dipolar plasmonic modes of different origins is possible under distinct combinations of many parameters. On the other hand, the electric field of the $s$-polarized light has one component perpendicular to the chain axis at any angle of incidence, and hence, only one plasmonic band of transversal character is expected. In the case of unpolarized light, the spectra are calculated as an average over the two polarizations directions, and the spectrum exhibits all LSPR modes.

In all the simulations, the interdipole separation was kept constant at around $0.53 \mathrm{~nm}$, giving a total of 14330 dipoles per nanosphere. The chosen number of dipoles was sufficient to mimic the structure of the nanosphere correctly and to achieve a proper convergence of the simulations. 


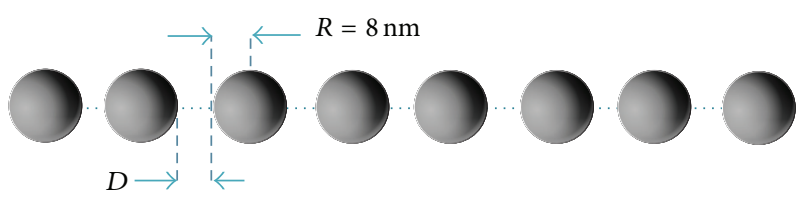

(a)

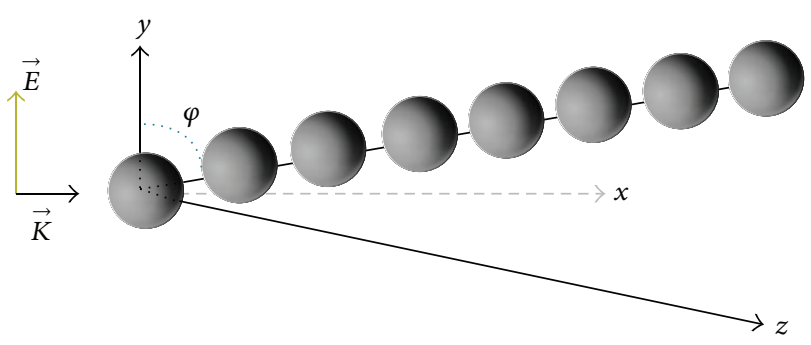

(b)

FIGURE 1: Schematic representation of a linear chain composed of 8 silver spheres (diameter $2 R$ ) with an interparticle separation of $D$. The incident electric field is parallel to the $y$-axis; $\varphi$ is the polarization angle between the electric field and the chain axis.

\section{Results and Discussion}

4.1. Chain Size. Assuming a dipolar approximation, the nanoparticles can be represented as point dipoles. In this sense, the interactions between them are classified into two types: (1) when $D \ll \lambda_{\text {inc }}$, the near-field interactions is dominant with a distance dependence of $1 / D^{3} ;(2)$ when $\lambda_{\text {inc }} / n_{m} \approx$ $D$, the plasmonic coupling is dominated by far-field interactions with a distance dependence of $D^{-1}$. The aim of the present study is to investigate the near-field plasmon coupling, without including any complications from the radiative coupling effect. The near-field coupling between nanoparticles within the chain leads to a pronounced change in the spatial distribution of the local field around the nanoparticles. This results in a significant change in the extinction spectra of the whole system. The optical behavior of interacting nanoparticles is no longer considered as the optical response of a single plasmonic entity but is rather considered as collective coupled resonances of the plasmons of all interacting bodies. For nanoparticles smaller in size, their extinction (absorption and scattering) spectrum is mainly dominated by the absorption cross-section [23]. This can be explained by the small albedo and the enhancement of the near-field absorption. Therefore, the optical spectra of the selected nanoparticles are considered to be represented by their absorption spectra.

It is necessary to represent the chain with a sufficient number of spheres, so that the plasmon parameters (resonance wavelength and plasmon bandwidth) become insensitive or independent of the chain size. To find a convenient $N$ that satisfies this criterion, several chains consisting of different $N$ s of identical spheres are considered. These nanospheres have a monotonic radius of $8 \mathrm{~nm}$ and are separated by $4 \mathrm{~nm}$ $(D=R / 2)$. It is assumed that the nanoparticles are embedded in polydimethylsiloxane (PDMS) $(n=1.46)$. The absorption spectra of the linear chain at different sizes are calculated under both parallel and perpendicular polarizations are shown in Figure 2.

When nanoparticles are placed in close proximity, the local fields of individual nanoparticles overlap, producing a plasmon coupling resonance. These coupled plasmon modes within the chain are caused by the electrostatic interaction between the opposite polarization charges on both sides of the gap separating neighboring nanospheres. This interaction extends from the nanoparticles surface to a distance comparable to their diameter [24]. In the target under investigation, the nanoparticles are placed close enough, so the plasmonic coupling is effective. The plasmon resonance wavelength is either red- or blue-shifted. The type of the plasmonic shift depends strongly on the direction of the incident electric field (incident polarization) [11]. The two scenarios will be discussed separately below in terms of $N$.

4.1.1. Parallel Polarization. At $\varphi=0^{\circ}$, the incident electric field is perfectly aligned along the chain axis which results in the charge oscillations in a direction parallel to that axis. This leads to the excitation of the longitudinal mode (LM). The resonance wavelength of the LM is red-shifted when the $N$ increases. The peak shifts from $425 \mathrm{~nm}$ for $N=2$ to $475 \mathrm{~nm}$ for $N=8$. The increase in the band position is accompanied by a slight increase in the absorption amplitude. The applied and induced electric fields are constructively combined. They are aligned in the same direction and against the Coulombic restoring force. The decrease in the static interaction results in an increase in the period of the electronic oscillations and, hence, the band position of the LM shifts to a higher wavelength as illustrated in Figure 2(a).

For longer chains $(N>8)$, the resonance wavelength becomes insensitive of the chain size with a significant enhancement in the absorption intensity. This enhancement indicates the dominant role of coupled plasmon modes in the chain. In a system of $N \geq 2$, the charges are accumulated at the opposite facing surfaces, thus greatly enhancing the local fields in the gaps between the nanoparticles. For this reason, these gaps are called hot spots and the combined local field amplification is called nanolensing $[13,25]$. These spots are localized at both the nanoparticles intersection points and along the line connecting them. Theoretical calculations and experimental measurements of the nanolensing are of great interest because they provide a way to amplify the spectroscopic signal of the adsorbed molecules in the vicinity of the nanoparticles.

The field enhancement is mainly controlled by damping processes of different origins. These processes are classified into radiative and dissipative damping. Due to the small size of the nanoparticles considered in the current study, the decrease in the peak enhancement with increasing $N$ caused by the radiation damping process is negligible. The increase in the absorption amplitude for larger numbers of $N$ has been observed experimentally [13]. Therefore, the theoretical results presented in this work are in agreement with the experimental observations. Wang et al. [13] studied the near-field coupling between spherical silver nanoparticles of 


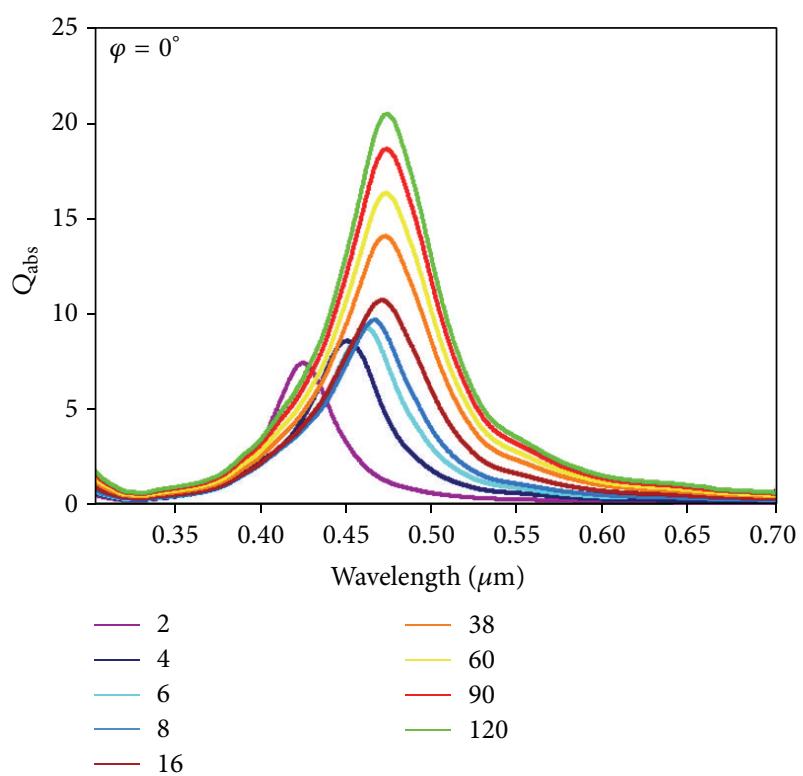

(a)

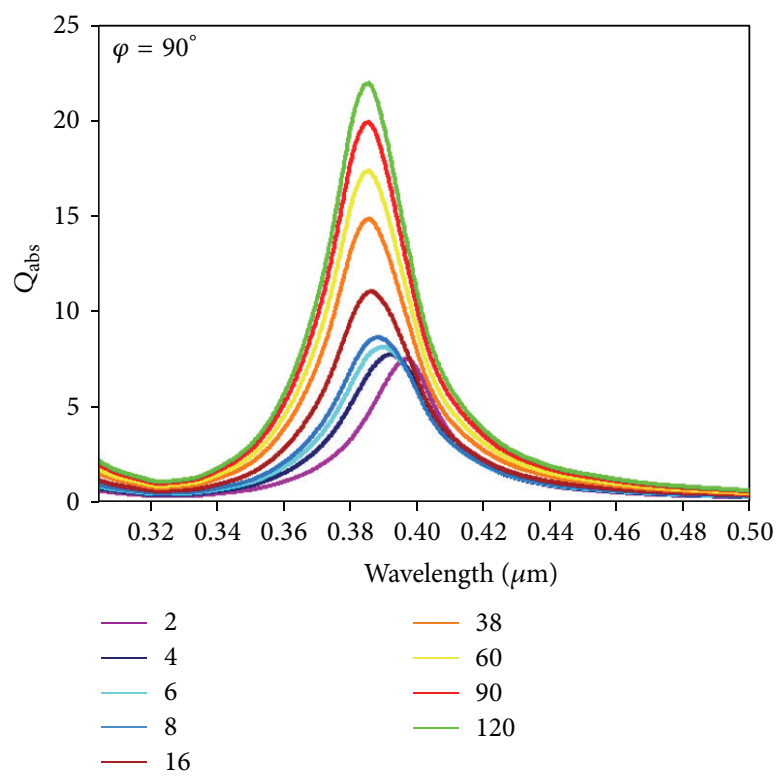

(b)

Figure 2: Absorption spectra of linear chains composed of various numbers of silver spheres under (a) parallel polarization and (b) perpendicular polarization.

a larger size $(R=20 \mathrm{~nm})$ in terms of the chain size. The nanoparticles were arranged in a close-packed linear chain. The nanoparticles were placed very close to each other at a distance of $1 \mathrm{~nm}$. The main finding in their study is that the field enhancement decreased with $N$ which contradicts both previously published experimental results and our current observations. Actually, the selected nanoparticles size is the key for explaining this contradiction. In the case of Wang's study, the nanoparticles were larger and as such, it is expected that the scattering cross-section has the main contribution to the extinction cross-section. This results in an enhancement of the radiation damping process as the size of the chain increases and, hence, decreases the amplitude of the field enhancement.

4.1.2. Perpendicular Polarization. Under perpendicular polarization, the electric field is aligned perpendicular to the chain axis. Therefore, another plasmonic band of different dipolar character is excited (transverse mode (TM)). In this case, the induced electric field is antiparallel (out of phase) to the applied one and it is in the same direction of the restoring force, thus increasing the resonance frequency (blue shift) as shown in Figure 2(b). The change in the band position of the LM is more noticeable as compared to that of the TM. This can be explained by the strong electrostatic interactions between the induced dipoles when the LM is excited. In both polarizations, as $N$ exceeds 8 nanoparticles, no change in the peak position of either LM or TM is observed because the plasmonic coupling reaches a saturation limit. For this reason, as well because of the satisfactory convergences and the reasonable computational costs, the chain consisting of 8 spheres is chosen to be representative of what will be studied in the subsequent sections. Anyhow, adding more nanoparticles into the chain increases the intensity of the induced field and, hence, the absorption efficiency with no change in the resonance energy of the plasmonic band.

4.2. Effect of the Dielectric Environment on Near-Field Interactions. In order to better understand how the plasmonic coupling influences the plasmonic modes, we compare the absorption spectra of silver linear chains when the nanoparticles $(2 R=8 \mathrm{~nm})$ are embedded in various environments $\left(n_{m}=1,1.33,1.46\right.$, and 1.66; the spectra corresponding to the last two values of $n_{m}$ will be omitted) at different $D s(0.2 R$, $0.25 R, 0.5 R, R, 2 R$, and $3 R)$. The dependency of the plasmon couplings on these parameters is studied at various polarization angles.

The absorption spectrum of a single nanosphere is shown in each set of calculated spectra for the linear chains. It exhibits a peak at $355 \mathrm{~nm}$ (in air) which represents the wellknown Mie's resonance in the quasistatic regime for an isotropic homogenous nanoparticle of spherical shape. Due to the high symmetry order of spherical nanospheres, their optical spectra are independent on both the polarization angle and polarization state of the incident light.

4.2.1. Parallel Polarization. At parallel polarization, the band position of the LM is blue-shifted with $D$ in all media. Due to enhancement of the coupled field, the LM is broadened in all media when nanoparticles are placed at distance smaller than $R$. At $D \leq R / 4$, in addition to the main plasmonic band, a shoulder at lower wavelength can be observed. While the main resonance peaks are due to the plasmonic coupling effect within the chain, the shoulder is associated with a dipole resonance of each single nanosphere [13]. In a water medium, the shoulder is located at a wavelength of around $380 \mathrm{~nm}$ with no significant change in either its band position or amplitude. 


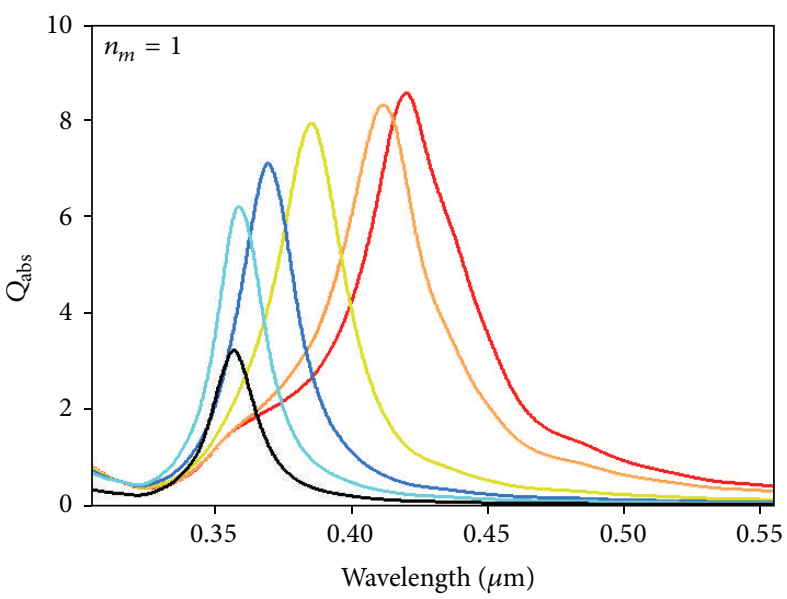

(a)

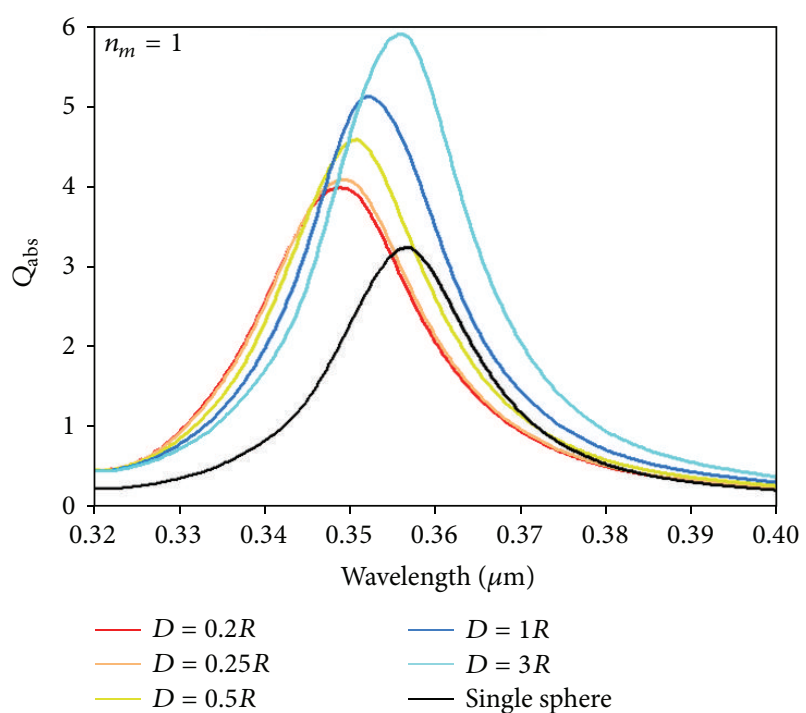

(c)

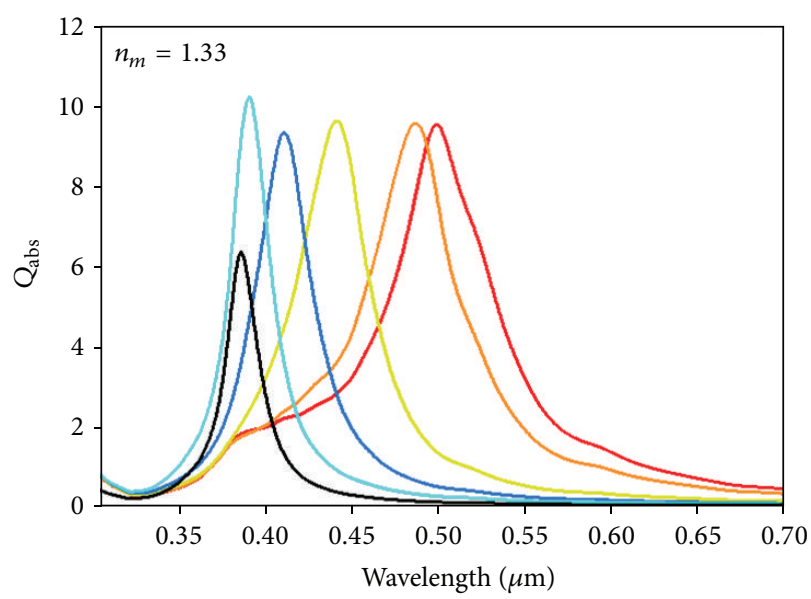

(b)

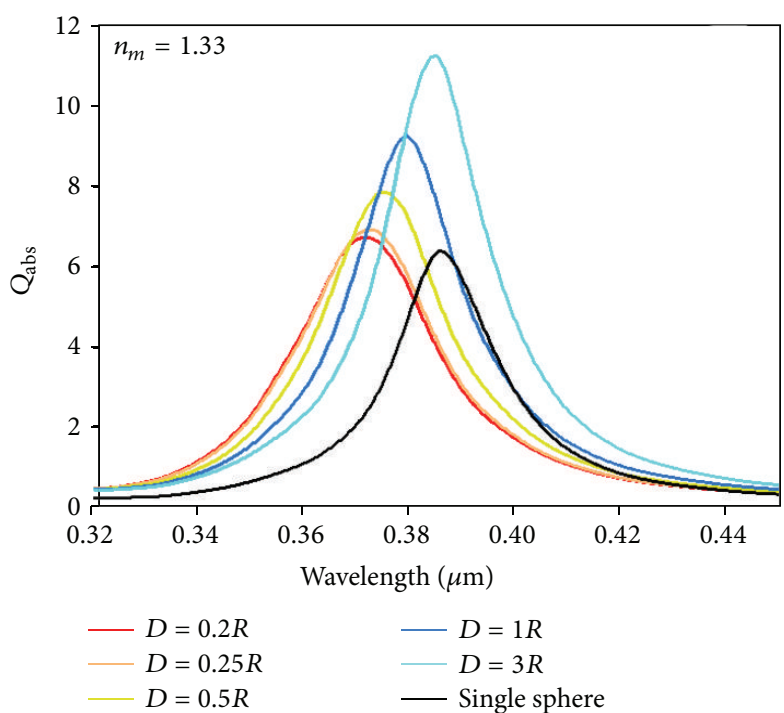

(d)

Figure 3: Absorption spectra of silver linear chains under $(a, b)$ parallel polarization and $(c, d)$ perpendicular polarization, at different interparticle separations and immersed in various embedding materials.

4.2.2. Perpendicular Polarization. When the incident field is transverse to the chains, the interactions between the nanoparticles are weak and cause a change in the resonance wavelength with $D$. The resonance wavelength of the TM is red-shifted as $D$ increases as illustrated in Figures 3(c) and $3(\mathrm{~d})$. In the case of perpendicular polarization, in a host media composed of air, the absorption efficiency of nanoparticles increases with $D$, in contrast to the case when the nanoparticles are illuminated by light of parallel polarization (see Figures 3(a) and 3(b)). In both polarizations, the band position of both TM and LM is red-shifted with $n_{m}$, with an increase in the absorption amplitude, as expected.

In an air environment, the absorption efficiency of the LM reaches a maximum around 8.6 at the smallest $D(=0.2 R)$ and then gradually decreases as $D$ increases. On the contrary, the amplitude of the TM increases with $D$. For $D \leq R$, the LM amplitude tends to have the same value as the surrounding medium becomes denser as shown in Figure 4(a). As the nanoparticles are set apart at a distance larger than the size of an individual sphere $(D=3 R)$, a sudden increase in the LM amplitude is observed. On the other hand, the TM amplitude is directly proportional to $n_{m}$ at all values of $D$ as illustrated in Figure 4(b).

In general, as $D$ increases, the type of the plasmon shift of both modes is in sharp contrast for both polarization directions. The diversity in their optical response can be explained on the basis of the dipole-dipole coupling model. Under parallel polarization, the coupling is attractive, while in the other polarization case, the coupling is repulsive. This results in red and blue shift in the plasmonic bands as $D$ decreases for parallel and perpendicular polarization, respectively. It is clear that the effect of the electromagnetic 


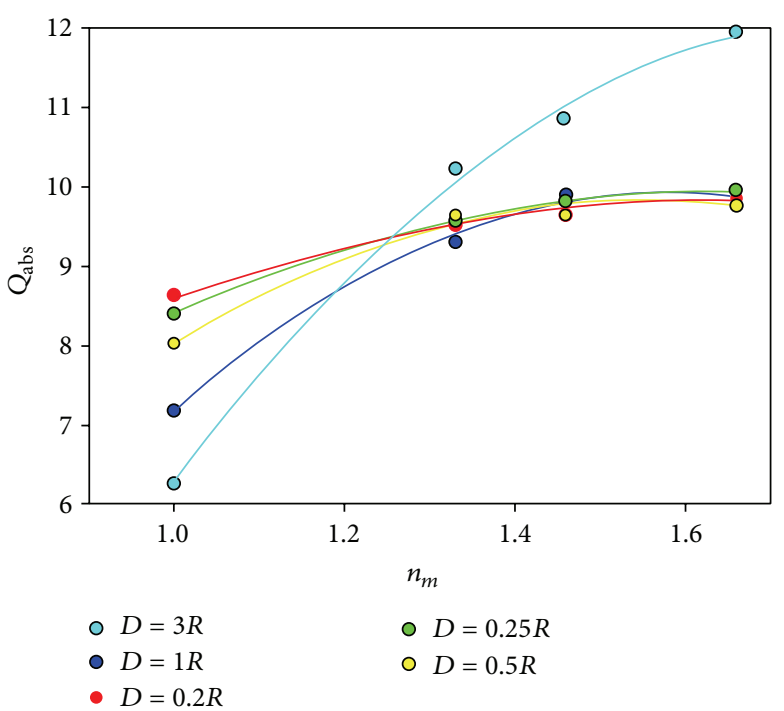

(a)

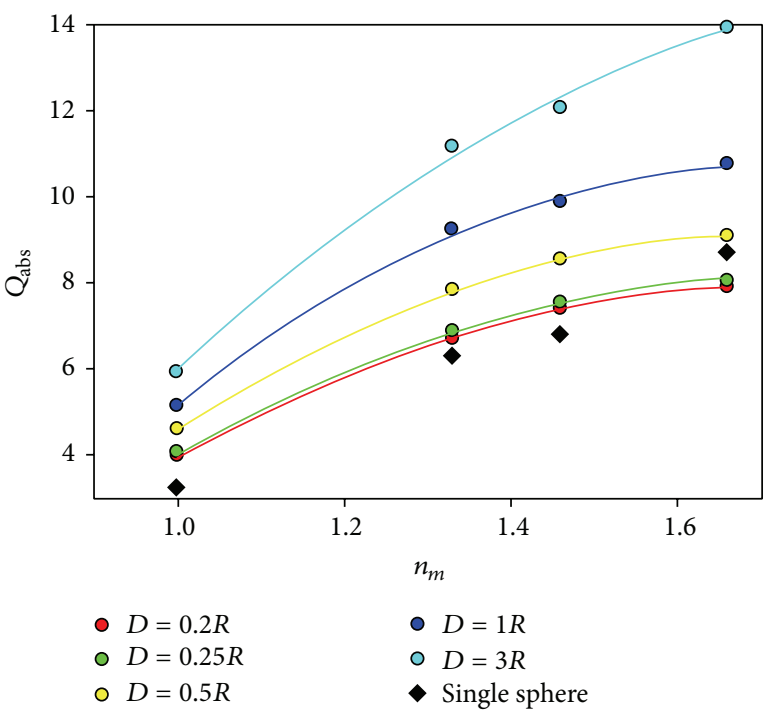

(b)

FIGURE 4: Absorption efficiency of various chains at different $D$ s as a function of $n_{m}$ for (a) parallel and (b) perpendicular polarizations.

coupling is stronger for the parallel polarization, as indicated by the large change in the band position of the coupled resonance mode.

In the case of dimers, the plasmonic couplings cause a shift in the resonance band that decays exponentially with D. Jain et al. [17] have derived a universal plasmon scaling behaviour, according to the so-called plasmon ruler equation. This equation can be exploited to estimate the interparticle separation in a metallic dimer:

$$
\frac{\Delta \lambda}{\lambda_{\mathrm{o}}}=\frac{\lambda_{\mathrm{res}}-\lambda_{\mathrm{o}}}{\lambda_{o}} \approx k \exp \left(\frac{-D / 2 R}{b}\right)
$$

where $\lambda_{\text {res }}, \lambda_{o}$, and $b$ are the fractional plasmon shift, the coupled resonance wavelength, the resonance wavelength of the single nanosphere, and the decay constant, respectively. The value of the constant $k$ depends on the type of the incident polarization. $k$ is positive for parallel polarization which indicates the shift of the LM to the higher wavelength. The negative value of $k$ is associated with perpendicular polarization and it indicates the blue-shift of the TM. In general, $k$ may be used to compare the amount of the plasmon shift; it exhibits larger magnitudes for the silver nanoparticles as compared to that of gold nanoparticles [17]. This indicates that, for silver nanoparticles, the local fields are stronger than the corresponding ones for gold. The interband transitions in the gold nanoparticles occur in the vicinity of the plasmon modes. The excitation of the interband electrons results in damping the resonance modes and hence, reduces the intensity of the local fields compared to that of silver nanoparticles.

As a result of the plasmonic coupling, it has been found that the lattice coherent frequency is also shifted. The change in the phonon frequency decays exponentially with $D$. The similar trend of both plasmon and phonon frequencies is
TABLE 1: Values of both constants $k$ and $b$ in terms of $n_{m}$ for parallel polarization.

\begin{tabular}{ccccc}
\hline & $n_{m}=1$ & $n_{m}=1.33$ & $n_{m}=1.46$ & $n_{m}=1.66$ \\
\hline$b$ & 0.185 & 0.206 & 0.221 & 0.235 \\
$k$ & 0.2904 & 0.4487 & 0.4881 & 0.552 \\
\hline
\end{tabular}

TABLE 2: Values of both constants $k$ and $b$ in terms of $n_{m}$ for perpendicular polarization.

\begin{tabular}{ccccc}
\hline & $n_{m}=1$ & $n_{m}=1.33$ & $n_{m}=1.46$ & $n_{m}=1.66$ \\
\hline$b$ & 0.933 & 0.5104 & 0.45135 & 0.4274 \\
$k$ & -0.0268 & -0.0427 & -0.0525 & -0.0647 \\
\hline
\end{tabular}

a result of dependency of the general scaling behavior on the field coupling.

The fractional plasmon shift in silver chains embedded in all the selected host exhibits the same universal decay trend as shown in Figure 5 (data are shown for only $n_{m}=$ 1.66). Therefore, the previous equation is still applicable for the multibody interaction problem considered in the current study.

Tables 1 and 2 show the dependency of both $k$ and $b$ on the dielectric properties of the host media for parallel and perpendicular polarizations, respectively. It seems that the value of $b$ is only slightly dependent on $n_{m}$ for parallel polarization, while the corresponding value for the perpendicular polarization is decreasing significantly with $n_{m}$. This means that the coupled TM decays faster than the corresponding LM. This discrepancy originates from the difference in the sensitivity of both modes to the change in the surrounding dielectric medium. The less sensitive the plasmonic mode is to $n_{m}$, the faster it will decay with $n_{m}$ [18]. For the LM, the change in the band position with $n_{m}$ is more pronounced 


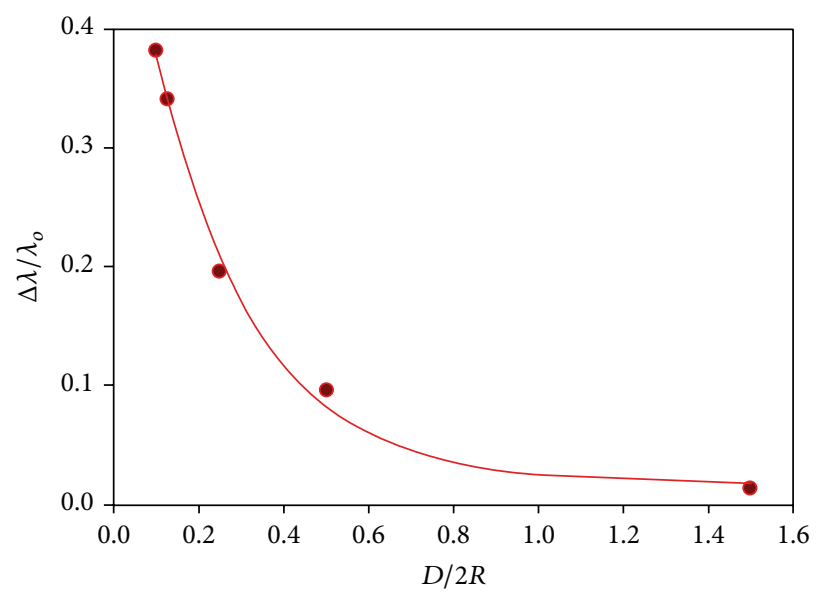

(a)

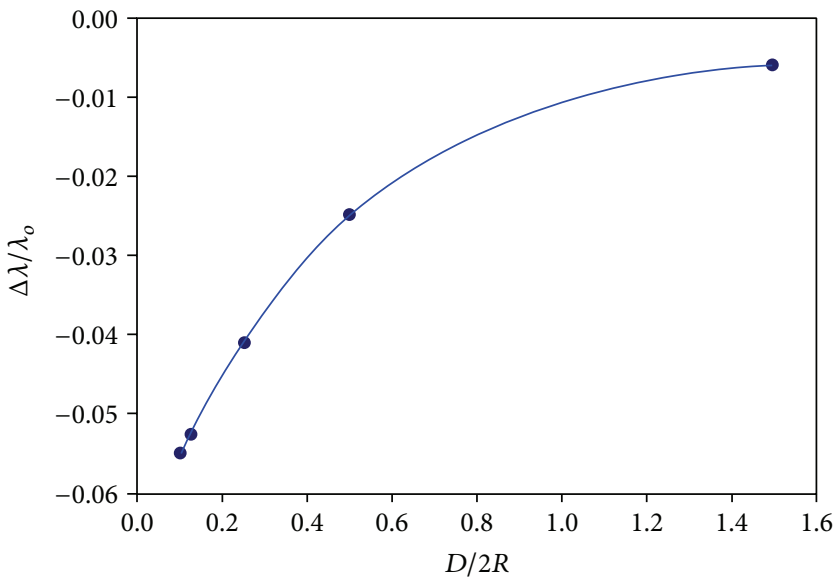

(b)

FIGURE 5: Fractional plasmon shift of the silver chains as a function of $D / 2 R$ at $n_{m}=1.66$ for (a) parallel and (b) perpendicular polarizations.

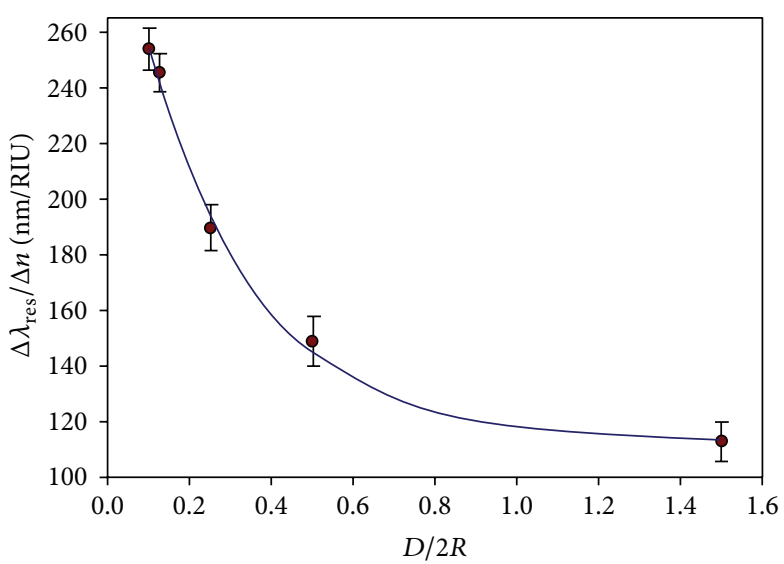

(a)

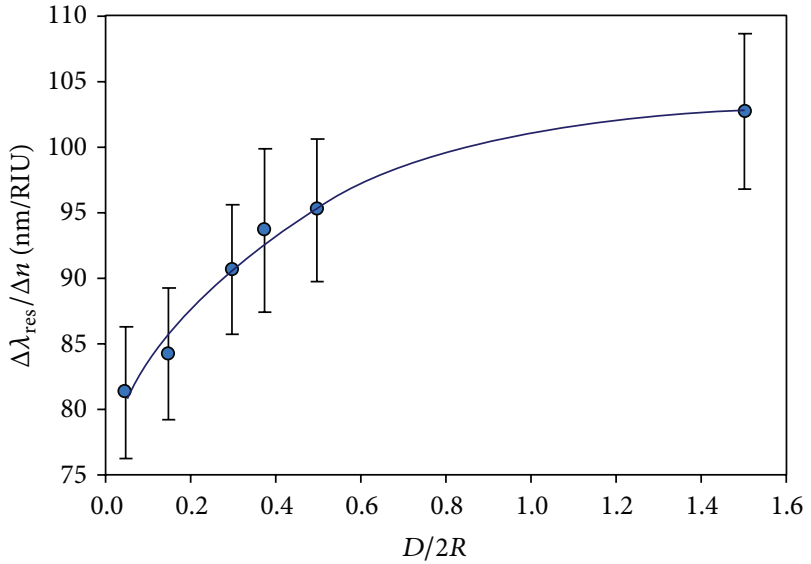

(b)

FIGURE 6: Plasmon resonance sensitivity of a silver nanoparticles chain to the surrounding medium as a function of the interparticle separation in units of sphere diameter at (a) parallel and (b) perpendicular polarizations.

compared to that of the TM, which can be indicated by the difference in the $k$ value. While the value of $k$ shows how sensitive the plasmonic mode is to $n_{m}$, the sign of $k$ shows the type of the plasmonic shift. A positive value of $\mathrm{k}$ indicates the red shift of LM and the negative value shows the blue shift of the TM. Jain and El-Sayed [18] found that the shift in the resonance increases in a denser medium (larger dielectric constant). The previously published results are in an agreement with our current observations.

Jain and El-Sayed also found that the value of $b$ for a system composed of gold nanodisc dimer is approximately equal to 0.2 in units of nanoparticles size for a polarization parallel to the interparticle axis. The $b$ value is independent of the nanoparticle shape, metal type, and the dielectric properties of the surrounding medium. This conclusion does not apply in our case; the value of $b$ is strongly dependent on both the dielectric properties of the surrounding medium and the polarization state of the incident light. The $b$ of the LM slightly increases showing a linear trend with $n_{m}$ and it is less than the expected value based on his observations. In the case of the orthogonal polarization, the corresponding value of $b$ shows a more nonlinear (exponential) dependency on $n_{m}$.

The coupling resonance wavelength is red-shifted in a linear proportion with increasing $n_{m}$ at any value of $D$ (data not shown). The slopes of these linear proportions as function of $D / 2 R$ are plotted in Figure 6. The sensitivity of the plasmonic mode to any change in the surrounding medium is monitored by the shift of its prospective peak position $\left(\Delta \lambda_{\text {res }}\right)$ per Refractive Index Unit (RIU) as shown in Figure 6. The band position of both coupled plasmonic modes is redshifted linearly in a dense medium as compared to the one in the vacuum. Increasing the value of the dielectric function of the surrounding adjusts the resonance condition and results in the lowering of the restoring force (the columbic interaction). Hence, the resonance occurs at a lower frequency. The LM exhibits larger magnitudes of red shift at smaller 


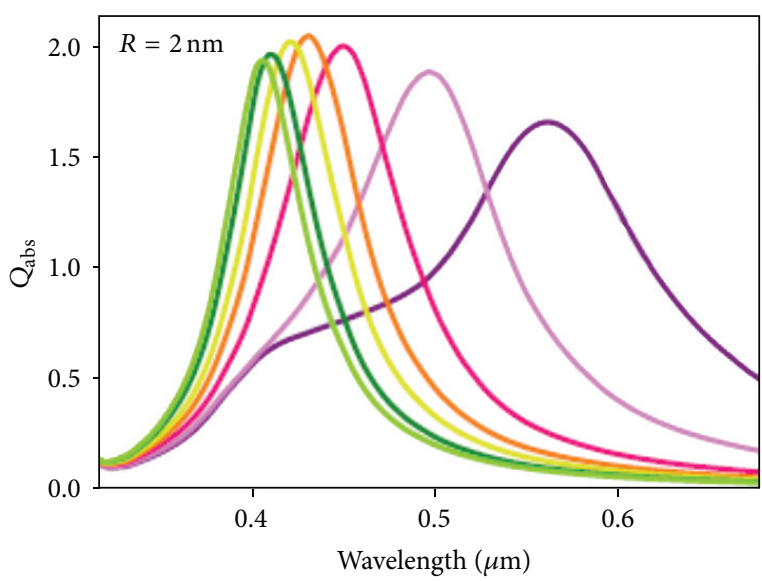

(a)

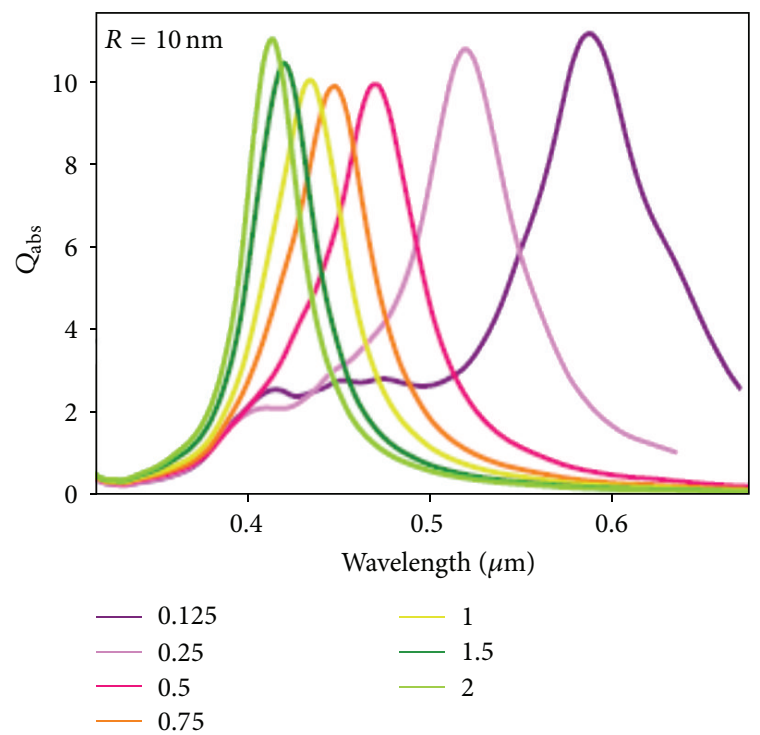

(c)

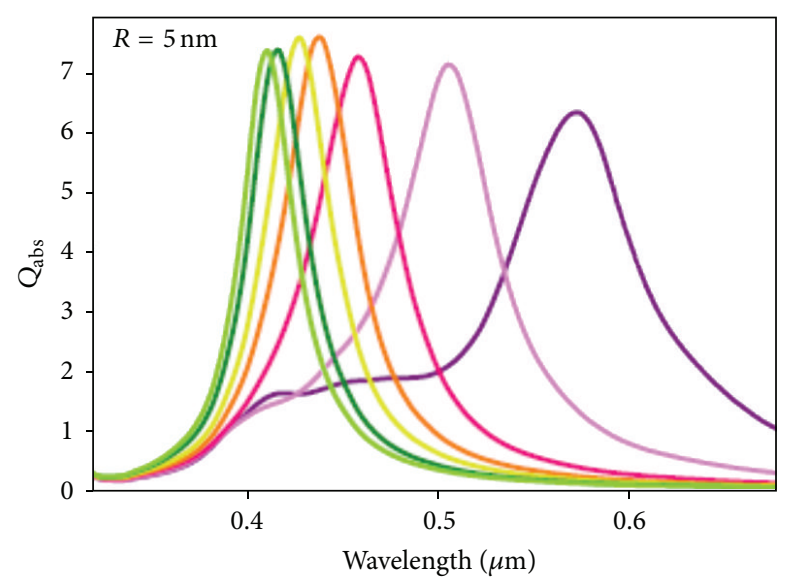

(b)

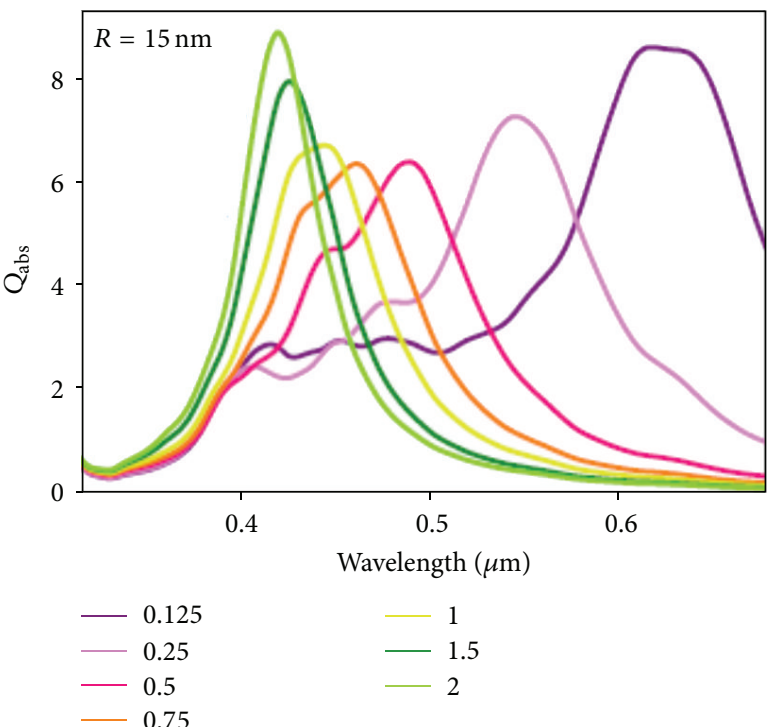

(d)

FIGURE 7: Absorption spectra of silver nanoparticles of different sizes arranged in linear chains under parallel polarization at different $D$ s (listed in the legends).

$D$ in response to an increment in the $n_{m}$. In other words, the sensitivity of the nanoparticles within the chain can be highly enhanced by decreasing $D$ under parallel polarization. In the case of parallel polarization, we found that the plasmon resonance is shifted exponentially with increasing $D / 2 R$ with $b=0.268$. Jain and El-Sayed [18] calculated the sensitivity of a $40 \mathrm{~nm}$ diameter gold nanosphere pair and found that it decays exponentially with increasing $2 D / R$ with $b=0.18$. The difference in $b$ originates from the diversity in both the nanoparticles size and the metal type.

On the contrary, at transversal excitation of the plasmonic mode, the sensitivity decays with decreasing $D / 2 R$ with $b=$ 0.43 as shown in Figure 6(b). The decay time $(\tau)$ is defined as reciprocal of $b$ and thus the sensitivity of the TM $(b=0.43)$ decays faster than the corresponding one of the LM $(b=$ 0.268). The diversity of the sensitivities between both modes is due to the difference in the field couplings. The strong plasmonic coupling under parallel polarization explains the higher sensitivity of the LM as compared to that of the TM.

\subsection{Effect of the Nanoparticles Size on Near-Field Interactions.} To investigate the effect of nanoparticle size on the nearfield couplings, a linear chain composed of eight identical spherical silver nanoparticles is simulated under both parallel and perpendicular polarizations. The radius of the spheres ranges between $2 \mathrm{~nm}$ and $15 \mathrm{~nm}$, at interparticle separations ranging between $D=R / 8$ and $D=2 R$. The $n_{m}$ is set to that of PDMS. The absorption spectra of silver chains at $\varphi=0^{\circ}$ and $90^{\circ}$ are plotted, respectively, in Figures 7 and 9.

4.3.1. Parallel Polarization. The profile lines of the absorption spectra are smooth and streamlined as long as the electric field is uniform across the silver nanoparticles. By increasing the particle size and decreasing $D$, the phase retardation effect 


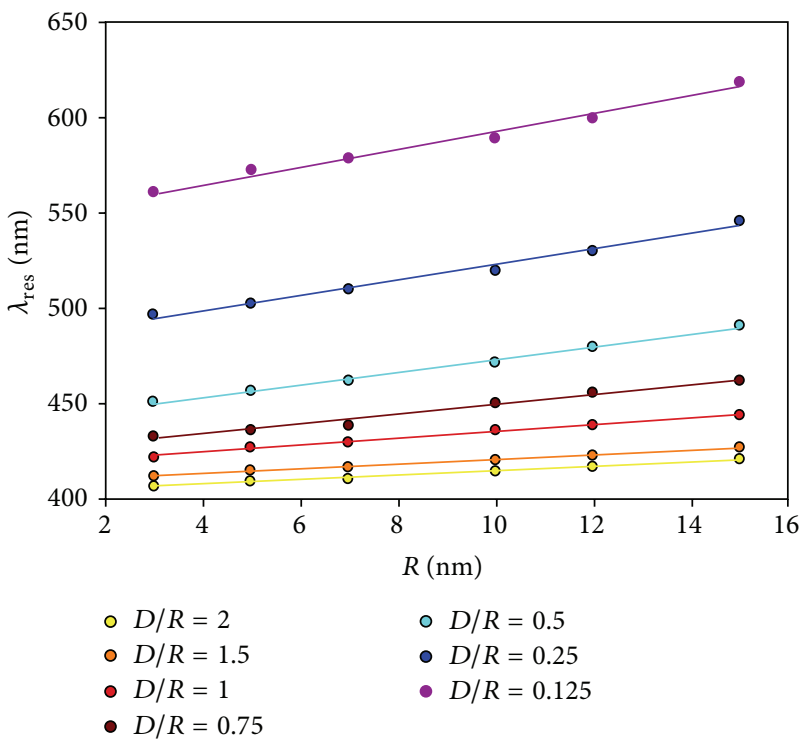

(a)

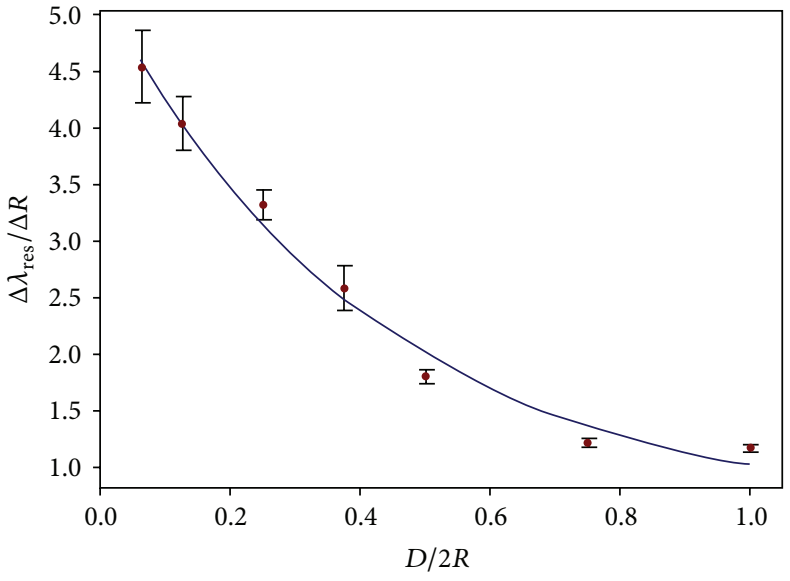

(b)

FIGURE 8: (a) LM of linear chains at different $D$ s (listed in the legend) as a function of $R$. (b) The change in the resonance wavelength of the LM is red-shifted linearly with $R$ at all $D$ s.

becomes significant. Nanoparticles of larger size experience this effect and it yields the excitation of plasmonic modes of quadrupole character. The quadrupole peaks are excited between 450 and $500 \mathrm{~nm}$ with relatively small amplitudes as shown in Figures $7(\mathrm{c})$ and $7(\mathrm{~d})$. As stated earlier, the excited plasmonic band observed at $400 \mathrm{~nm}$ represents Mie's resonance of the individual sphere. Eventually, increasing the component particles size, the plasmon coupling absorption bands became deformed and deviated from the Gaussian bell shape.

The coupled resonance wavelength (LM) is linearly redshifted with $R$ for any value of $D$ as shown in Figure 8(a). The slope of the straight lines represents the sensitivity of the LM to any change of $R$. To further investigate the sensitivity of the band position of the LM to the $D$ in terms of $R$, the ratio $\Delta \lambda_{\text {res }} / \Delta R$ has been calculated at various $D$ s. It is found that this ratio decays exponentially ( $b=0.413$ and $a=4.657$ ) with $D / R$ as shown in Figure $8(\mathrm{~b})$. As $D / R$ decreases from 1 to 0.12 , the sensitivity $\left(\Delta \lambda_{\text {res }} / \Delta R\right)$ increases up to $4.54 \mathrm{~nm}$ per $\mathrm{nm}$ change in $R$. Thus, the dependency of the plasmonic energy of the LM on nanoparticles size is more pronounced at smaller $R$ and, especially, at smaller $D$. This result is in contradiction with the universality of the plasmon ruler law derived by Jain et al. [17], whereas it agrees with Encina and Coronado's work [26] illustrating that, in a system, composed of silver nanosphere pairs, the fractional plasmon shift depends on the size of the nanoparticle pair.

4.3.2. Perpendicular Polarization. At $\varphi=90^{\circ}$, the excited plasmonic band has a transversal character. The resonance wavelength of the TM is blue-shifted with decreasing $D$ at all values of $R$. The absorption spectra calculated for different $R \mathrm{~s}$ are plotted in Figure 9.
The result of the simulations shows that the absorption spectrum of an individual nanosphere depends strongly on its size. Upon increasing the size, it is found that the absorption efficiency linearly increases as $R$ increases until it reaches a maximum value at $R=13 \mathrm{~nm}$ as shown in Figure 10(a). In the case of the nanoparticles arranged in $1 \mathrm{D}$ arrays, the coupling energy reduces the optimum size $\left(R_{0}\right)$ corresponding to the maximum absorption. The value of $R_{0}$ of an isolated sphere is reduced in the chain from $13 \mathrm{~nm}$ to $12 \mathrm{~nm}, 8 \mathrm{~nm}$ for perpendicular and parallel polarizations, respectively. When $R_{0}>$ $R$, the extinction efficiency has mainly contributions from the scattering cross-section resulting in decreased absorption amplitude as shown in Figure 10(a). The phase retardation effect is not obvious in the absorption spectra of the TM. It seems that the quadrupole modes are excited in the vicinity of the TM, making them indistinguishable because of their small relative amplitudes to the dominant TM.

The sensitivity of the transversal coupled resonance to $R\left(\Delta \lambda_{\text {res }} / \Delta R\right)$ as a function of the dimensionless quantity $D / 2 R$ is shown in Figure 10(b). It is found that $\Delta \lambda_{\text {res }} / \Delta R$ decreases linearly with increasing $D$ and that it does not obey the universal scaling behavior.

\section{Conclusion}

In summary, we have examined (the generalization of) the universal scaling behavior for spherical silver nanoparticles arranged in finite linear chains (eight nanoparticles) and embedded in different host media. The results of simulation show that the plasmon ruler equation can be successfully extended to represent the fractional plasmon shift of many interacting nanoparticles. The decay length and the mount of the fractional shift of the silver linear chains strongly 


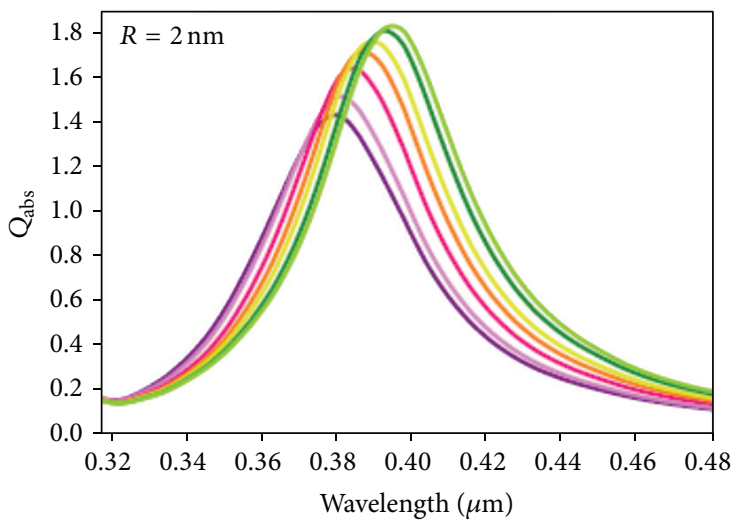

(a)
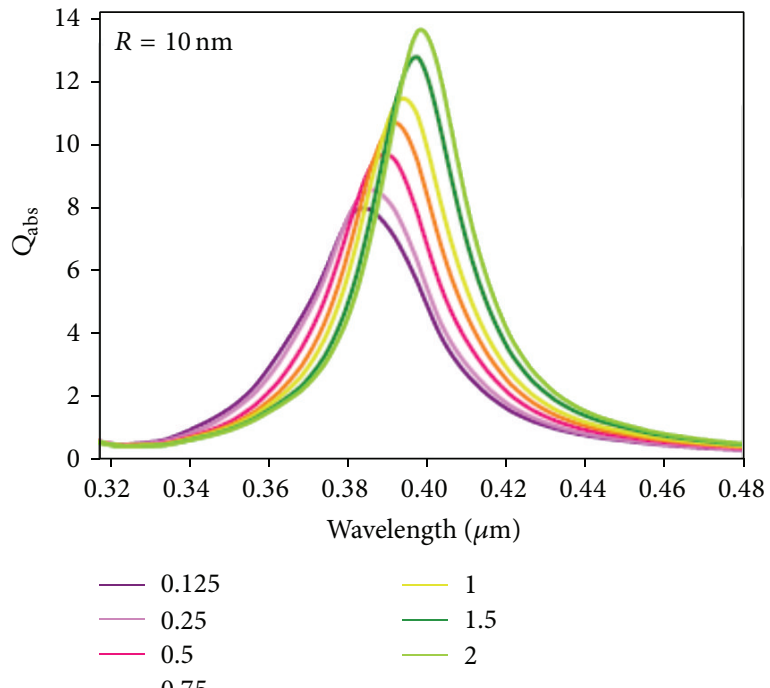

(c)

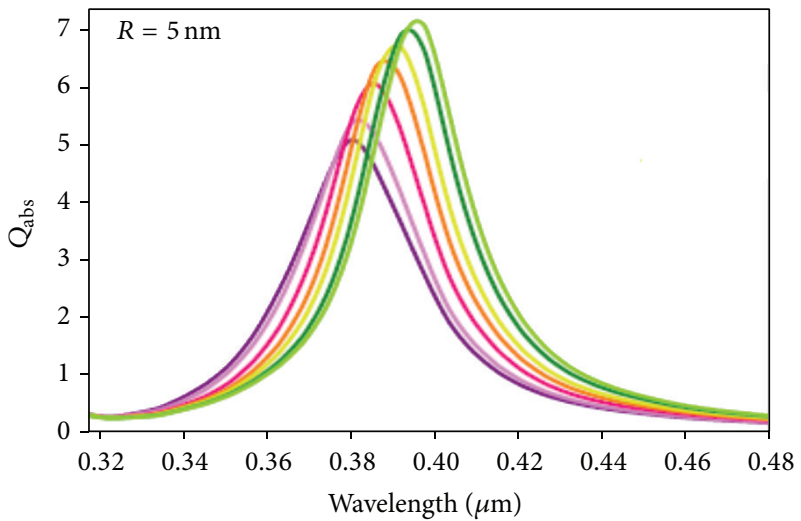

(b)

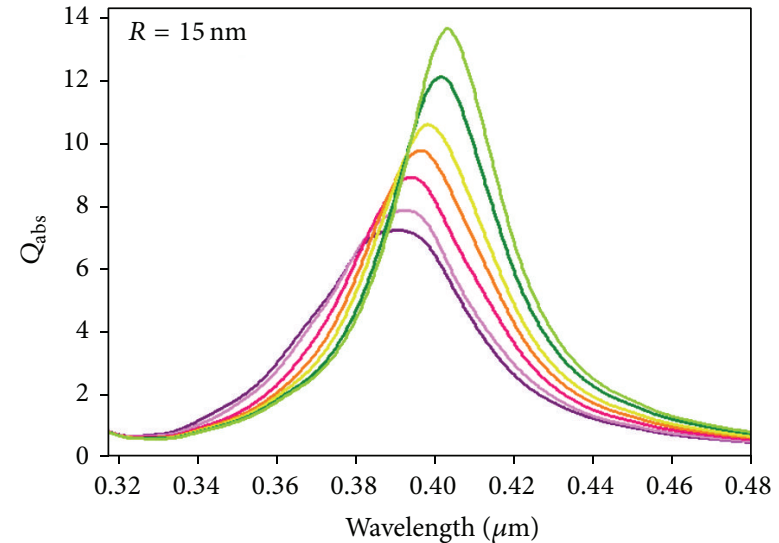

(d)

FIGURE 9: Absorption spectra of silver nanoparticles of different sizes and arranged in linear chains under perpendicular polarization at different $D$ s (listed in the legends).

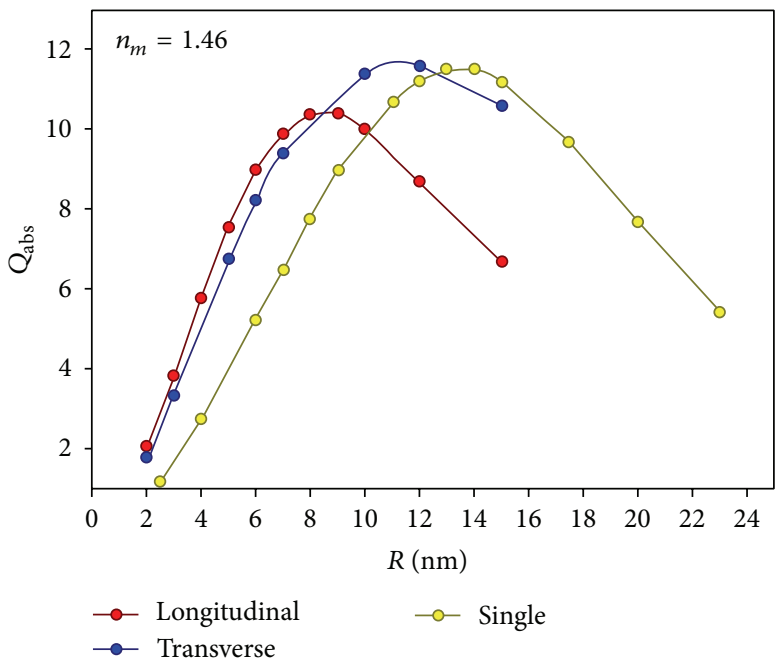

(a)

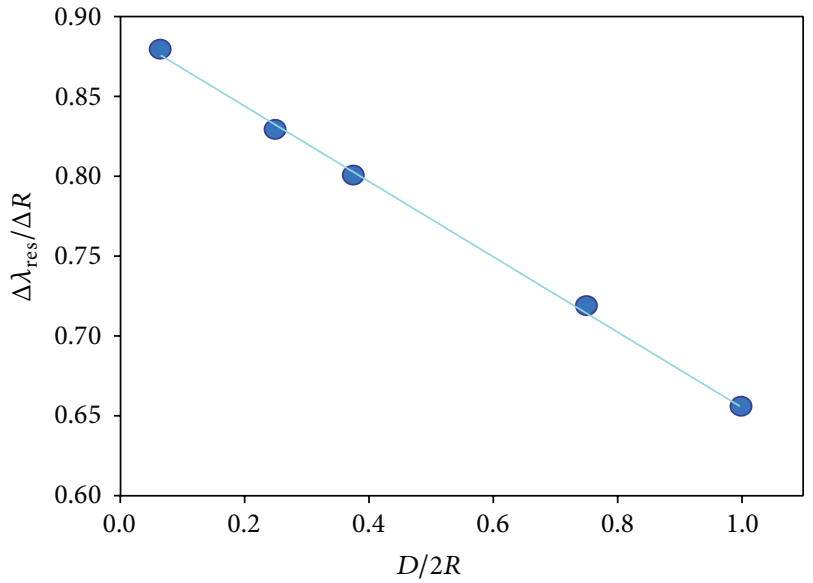

(b)

Figure 10: (a) Absorption efficiency of the dipole plasmon resonance as a function of $R$ for (i) an individual silver nanoparticles (yellow) and (ii) a linear silver chain at the $D=R$ (under parallel polarization in red and under perpendicular polarization in blue). (b) Sensitivity of the TM to $R\left(\Delta \lambda_{\text {res }} / \Delta R\right)$ as a function of $D / 2 R$. 
depend on the polarization state of the incident polarization. The LM exhibits a significant shift of the plasmon resonance (indicated by the value of $k$ ) as compared to that of the TM. This can be explained by the fact that the enhancement of the local field under parallel polarization is more pronounced. On the other hand, the decay of the TM shows a strong dependency on the dielectric function of the surrounding medium. It decays twice faster than the LM, as the host medium becomes denser.

The dependency of the universal scaling behavior on the nanoparticle size within the linear chains is also examined. The sensitivity of the both the coupled plasmonic modes (LM and TM) shows a strong dependency on the size of the interacting nanoparticles. The change in the longitudinal resonance wavelength with respect to the nanoparticles size $\left(\Delta \lambda_{\text {res }} / \Delta R\right)$ decays exponentially with $D / R$ and obeys the universal law. The dependency of the longitudinal plasmonic energy on nanoparticles size is more pronounced at smaller values of $R$ and $D$. The corresponding sensitivity of the TM changes linearly with $D / R$, which means that the universal law does not apply in this case.

\section{Conflict of Interests}

The authors declare that there is no conflict of interests regarding the publication of this paper.

\section{Acknowledgment}

This work has been supported by the Natural Sciences and Engineering Research Council of Canada (NSERC) which is gratefully acknowledged.

\section{References}

[1] Y. Fang, "Optical absorption of nanoscale colloidal silver: aggregate band and adsorbate-silver surface band," The Journal of Chemical Physics, vol. 108, no. 10, pp. 4315-4318, 1998.

[2] G. Schmid, Clusters and Colloids: From Theory to Applications, vol. 7, VCH, Weinheim, Germany, 1994.

[3] M. Alsawafta, M. Wahbeh, and V.-V. Truong, "Simulated optical properties of gold nanocubes and nanobars by discrete dipole approximation," Journal of Nanomaterials, vol. 2012, Article ID 283230, 9 pages, 2012.

[4] M. Alsawafta, M. Wahbeh, and V.-V. Truong, "Plasmonic modes and optical properties of gold and silver ellipsoidal nanoparticles by the discrete dipole approximation," Journal of Nanomaterials, vol. 2012, Article ID 457968, 10 pages, 2012.

[5] K. A. Willets and R. P. van Duyne, "Localized surface plasmon resonance spectroscopy and sensing," Annual Review of Physical Chemistry, vol. 58, pp. 267-297, 2007.

[6] E. Hutter and J. H. Fendler, "Exploitation of localized surface plasmon resonance," Advanced Materials, vol. 16, no. 19, pp. 1685-1706, 2004.

[7] J. N. Anker, W. P. Hall, O. Lyandres, N. C. Shah, J. Zhao, and R. P. Van Duyne, "Biosensing with plasmonic nanosensors," Nature Materials, vol. 7, no. 6, pp. 442-453, 2008.

[8] W. J. Galush, S. A. Shelby, M. J. Mulvihill, A. Tao, P. Yang, and J. T. Groves, "A nanocube plasmonic sensor for molecular binding on membrane surfaces," Nano Letters, vol. 9, no. 5, pp. 20772082, 2009 .
[9] B. Nikoobakht, J. Wang, and M. A. El-Sayed, "Surface-enhanced Raman scattering of molecules adsorbed on gold nanorods: offsurface plasmon resonance condition," Chemical Physics Letters, vol. 366, no. 1-2, pp. 17-23, 2002.

[10] R. F. Aroca, R. A. Alvarez-Puebla, N. Pieczonka, S. SanchezCortez, and J. V. Garcia-Ramos, "Surface-enhanced Raman scattering on colloidal nanostructures," Advances in Colloid and Interface Science, vol. 116, no. 1-3, pp. 45-61, 2005.

[11] A. O. Pinchuk and G. C. Schatz, "Nanoparticle optical properties: far- and near-field electrodynamic coupling in a chain of silver spherical nanoparticles," Materials Science and Engineering B: Solid-State Materials for Advanced Technology, vol. 149, no. 3, pp. 251-258, 2008.

[12] L. Zhao, K. Lance Kelly, and G. C. Schatz, "The extinction spectra of silver nanoparticle arrays: influence of array structure on plasmon resonance wavelength and width," The Journal of Physical Chemistry B, vol. 107, no. 30, pp. 7343-7350, 2003.

[13] Z. B. Wang, B. S. Luk'yanchuk, W. Guo et al., "The influences of particle number on hot spots in strongly coupled metal nanoparticles chain," The Journal of Chemical Physics, vol. 128, no. 9, Article ID 094705, 2008.

[14] B. Willingham and S. Link, "Energy transport in metal nanoparticle chains via sub-radiant plasmon modes," Optics Express, vol. 19, no. 7, pp. 6450-6461, 2011.

[15] T. R. Jensen, M. L. Duval, K. Lance Kelly, A. A. Lazarides, G. C. Schatz, and R. P. Van Duyne, "Nanosphere lithography: effect of the external dielectric medium on the surface plasmon resonance spectrum of a periodic array of silver nanoparticles," Journal of Physical Chemistry B, vol. 103, no. 45, pp. 9846-9853, 1999.

[16] A. J. Haes, S. Zou, G. C. Schatz, and R. P. van Duyne, "A nanoscale optical biosensor: the long range distance dependence of the localized surface plasmon resonance of noble metal nanoparticles," Journal of Physical Chemistry B, vol. 108, no. 1, pp. 109-116, 2004.

[17] P. K. Jain, W. Huang, and M. A. El-Sayed, "On the universal scaling behavior of the distance decay of plasmon coupling in metal nanoparticle pairs: a plasmon ruler equation," Nano Letters, vol. 7, no. 7, pp. 2080-2088, 2007.

[18] P. K. Jain and M. A. El-Sayed, "Noble metal nanoparticle Pairs: effect of medium for enhanced nanosensing," Nano Letters, vol. 8, no. 12, pp. 4347-4352, 2008.

[19] P. K. Jain and M. A. El-Sayed, "Surface plasmon coupling and its universal size scaling in metal nanostructures of complex geometry: elongated particle pairs and nanosphere trimers," The Journal of Physical Chemistry C, vol. 112, no. 13, pp. 4954-4960, 2008.

[20] B. T. Draine and P. J. Flatau, "Discrete-dipole approximation for periodic targets: theory and tests," The Journal of the Optical Society of America A, vol. 25, no. 11, pp. 2693-2703, 1994.

[21] M. J. Collinge and B. T. Draine, "Discrete-dipole approximation with polarizabilities that account for both finite wavelength and target geometry," Journal of the Optical Society of America A, vol. 21, no. 10, pp. 2023-2028, 2004.

[22] J. J. Goodman, B. T. Draine, and P. J. Flatau, "Application of fastFourier-transform techniques to the discrete-dipole approximation," Optics Letters, vol. 16, no. 15, pp. 1198-1200, 1991.

[23] P. K. Jain, K. S. Lee, I. H. El-Sayed, and M. A. El-Sayed, "Calculated absorption and scattering properties of gold nanoparticles of different size, shape, and composition: applications in biological imaging and biomedicine," Journal of Physical Chemistry B, vol. 110, no. 14, pp. 7238-7248, 2006. 
[24] C. Noguez, "Surface plasmons on metal nanoparticles: the influence of shape and physical environment," The Journal of Physical Chemistry C, vol. 111, no. 10, pp. 3806-3819, 2007.

[25] S. Foteinopoulou, J. P. Vigneron, and C. Vandenbem, "Optical near-field excitations on plasmonic nanoparticle-based structures," Optics Express, vol. 15, no. 7, pp. 4253-4267, 2007.

[26] E. R. Encina and E. A. Coronado, "Plasmon coupling in silver nanosphere pairs," Journal of Physical Chemistry C, vol. 114, no. 9, pp. 3918-3923, 2010. 

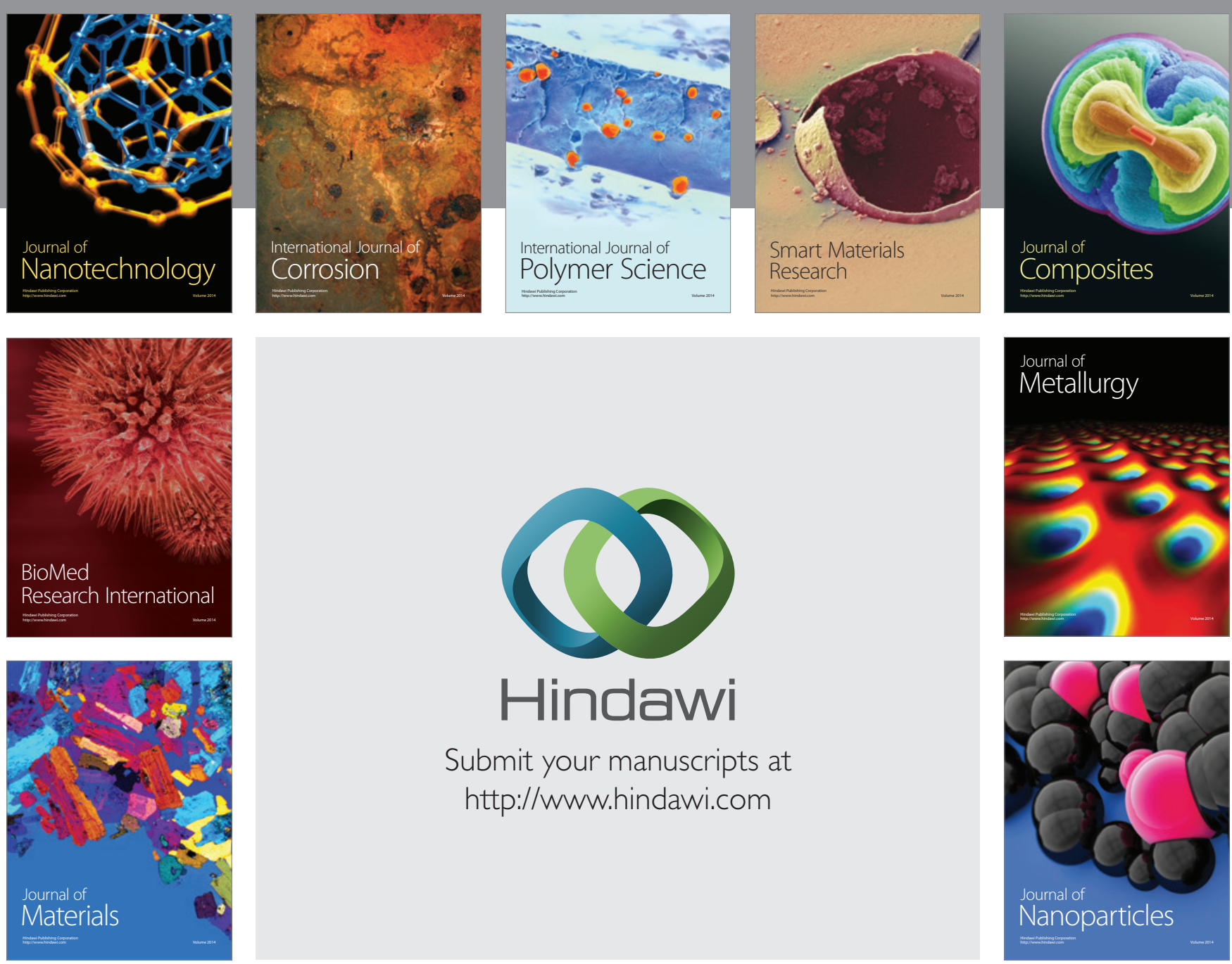

Submit your manuscripts at http://www.hindawi.com
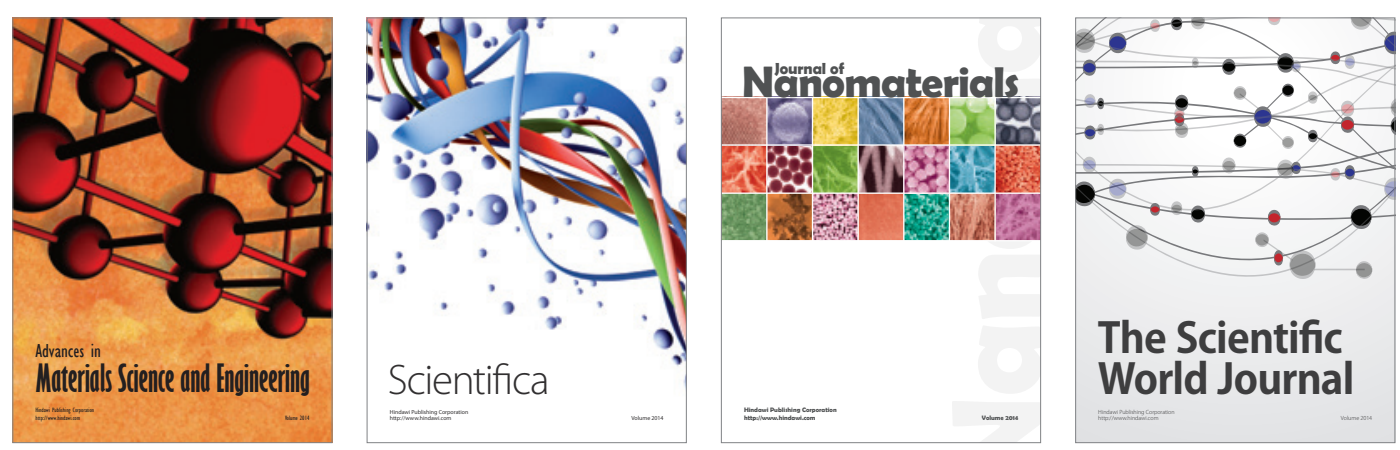

\section{The Scientific World Journal}
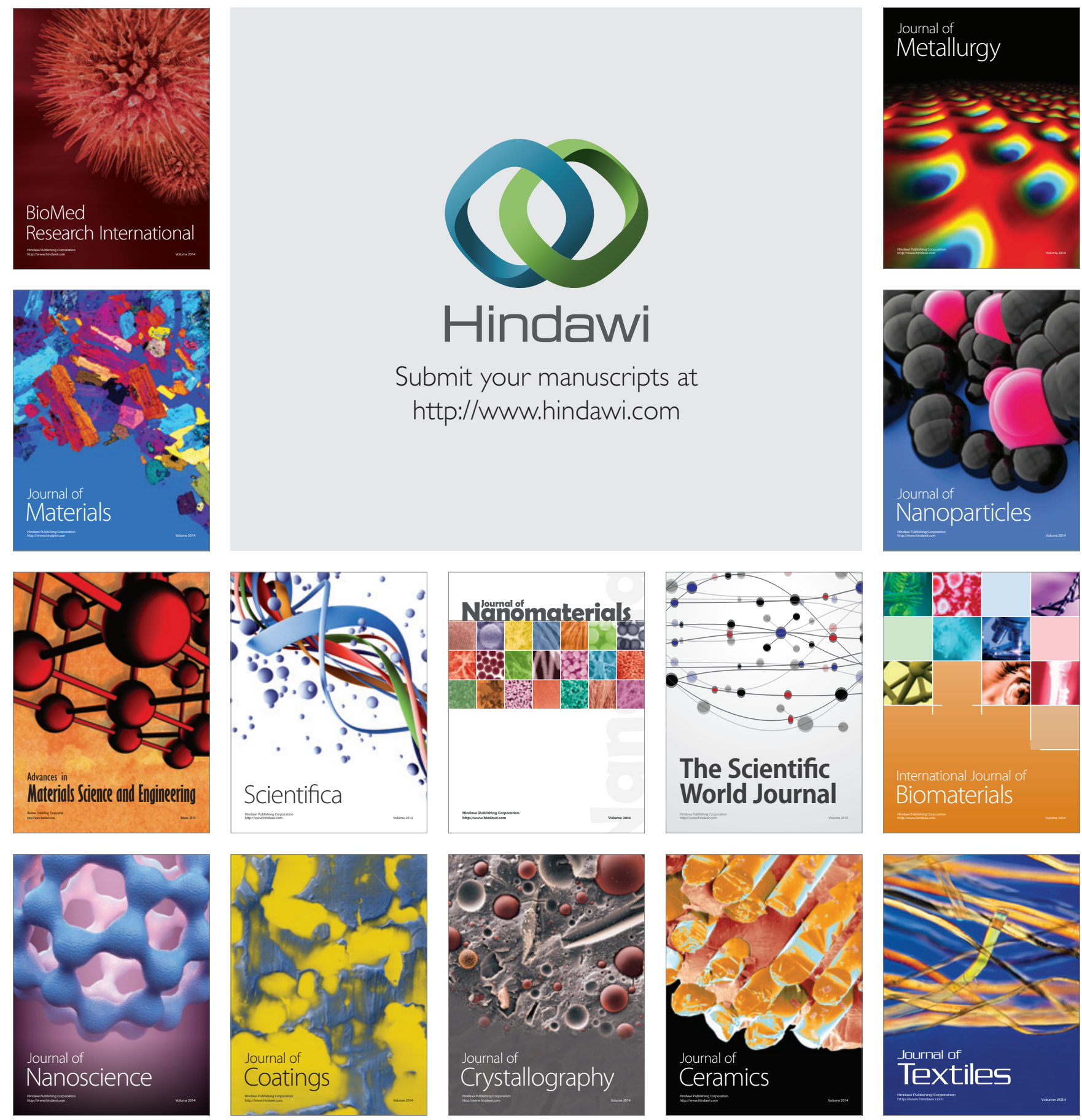